\title{
El idioma, como vehículo de la comunicación cien- tífica: análisis de la visibilidad e impacto de la in- vestigación a través de la bibliometría de Google
}

\author{
José Manuel De Pablos Coello \\ jpablos@ull.edu.es \\ Universidad de La Laguna \\ Miguel TúÑEZ LÓPEZ \\ miguel.tunez@usc.es \\ Universidad de Santiago de Compostela \\ Concha Mateos Martín \\ concepción.mateos@urjc.es \\ Universidad Rey Juan Carlos de Madrid
}

Recibido 24 de febrero de 2014

Aceptado: 22 de julio de 2014

\begin{abstract}
Resumen
La irrupción de Google en la bibliometría con indicadores personales (investigadores) y colectivos (revistas) ha sacudido las referencias de quienes operan con índices de impacto y con contabilización de citas, principalmente en Ciencias Sociales y Humanidades. Sus nueve listas de las 100 revistas más citadas según su lengua de publicación, actualizadas en 2012 y 2013, permiten iniciar análisis comparativos para valorar el peso del castellano en la difusión científica referenciados en los índices $h$ y $h 5$ de las revistas. Metodología: análisis de datos de Google Scholar a través de la media universal de h5-index $(\mu)$ y la media universal de h5-median $(\mu \mathrm{m})$ para conocer las áreas dominantes en el panorama internacional de la difusión investigadora, en el que se advierte un desequilibrio del peso internacional del castellano como soporte vehicular de ciencia.

Palabras clave: Google Scholar, revistas Top 100, lengua española, índice-h5, media universal de h5index, índice $\mu$, media universal de h5-median, índice $\mu \mathrm{m}$.

\section{Language as a Vehicle for the Dissemination of Science: Analysis of the Visibility and Impact of Research through Google Bibliometrics}

\begin{abstract}
The emergence of Google in the field of bibliometrics through personal and collective indicators (researchers and journals) has affected the references of those who rely on impact factors and the counting of citations, particularly within the social sciences and the humanities. Google's lists of the 100 most cited publications in nine different languages, which were updated in 2012 and 2013, allow us to carry out comparative analyses to assess the impact of the Spanish language in the dissemination of science based on the journals' $h$ and $h 5$ indexes. Method: this study is based on the analysis of Google Scholar data, particularly the universal h5-index $(\mu)$ and the universal h5-median $(\mu \mathrm{m})$, in order to identify the areas of the international dissemination of science in which the impact of the Spanish language is the most and least intense.

Keywords: Google Scholar, Top 100 journals, Spanish language, index-h5, universal h5- index, $\mu$ index, universal h5-median, $\mu \mathrm{m}$ index.

\section{Referencia normalizada}

DE PABLOS COELLO, José Manuel; TÚÑEZ LÓPEZ, Miguel; y MATEOS MARTÍN, Concha (2015): "El idioma, como vehículo de la comunicación científica: análisis de la visibilidad e impacto de la investigación a través de la bibliometría de Google". Estudios sobre el Mensaje Periodístico. Vol. 21, Núm. 1 (enero-junio), págs.: 283-298. Madrid, Servicio de Publicaciones de la Universidad Complutense.
\end{abstract}


Sumario: 1. Introducción. 2. Indicadores de $h: h 5, i 10, g, a$ y $h_{R}$. 3. Bibliometría en Google Scholar Metrics e impacto en las Ciencias Sociales. 4. El área de Comunicación: investigadores y revistas. 5. Metodología; 5.1. Población y muestra; 5.2. Objetivos e instrumentos; 5.3. Hipótesis. 6. Resultados; 6.1. Por idiomas; 6.2. Por áreas o campos de conocimiento. 7. Conclusiones. 8. Referencias bibliográficas.

\section{Introducción}

El índice $h$ ( $h$-index) ha modificado la forma de entender la visibilidad de la investigación porque acerca los indicadores bibliométricos a las esferas personales, ya que permite medir el impacto personal de los investigadores y no solo, como era habitual, el impacto colectivo de las revistas. En palabras de Hirsch (2005), un investigador tiene índice $h$ si el valor de su número de artículos $(N A)$ tiene al menos $h$ citas cada uno y los otros artículos $(N A-h)$ tienen menos de $h$ citas cada uno. Es decir, representa el número $h$ de publicaciones que han recibido por lo menos un número $h$ de citas. El propio Hirsch enunciaba que un indicador que puede aplicarse a las partes de un todo debe poder aplicarse también al todo en su conjunto, con lo que dejaba claro que la propuesta es un indicador para investigadores y para grupos de investigadores (Departamentos, centros de investigación, revistas, etc.).

La comunidad científica ha aceptado $h$ como indicador bibliométrico porque refiere a un mismo tiempo producción e impacto y porque se considera útil para evaluar la actividad de investigadores frente al factor de impacto, que evalúa revistas (Costas y Bordons, 2007; Imperial y Rodríguez, 2007; Grupo Scimago, 2006; Dorta y Dorta, 2010; Silva, 2012). Una forma de constatar esa aceptación es observar cómo Thomson Reuters (Web of Science) y Elsevier (Scopus) lo han incorporado a sus indicadores de impacto (Túñez, 2013). Desde 2012, Scopus y WoS tienen la competencia directa de Google Scholar Metrics (GSM) que elabora y publica listados del índice $h$ de revistas en varias lenguas y ofrece a los investigadores la posibilidad de crear su perfil para obtener sus índices $h, h 5$ e $i h 10$ personales.

Este trabajo analiza, con carácter exploratorio, el peso del idioma -globalmente y por áreas- como soporte de difusión científica tomando muestra cerca de 1.000 revistas en 2012 y casi 900 en 2013 en lenguas inglesa, china, portuguesa, alemana, española, francesa, italiana, japonesa, holandesa, todas ellas consideradas por GSM como las más citadas. La comparativa se realiza, básicamente, calculando la media universal $(\mu)$, definida como la media aritmética de los índice $h 5$ superior e inferior de las revistas integrantes de cada tabla de las 100 revistas más citadas en una lengua, para disponer de números más concretos, sencillos, unificados y comparables. Se interpretan indicadores disponibles agrupando las cabeceras en español en seis áreas: Salud, Ciencias Sociales, Ciencias de la Naturaleza, Humanidades, Ciencias y Tecnologías e Ingenierías.

\section{Indicadores de h: h5, i10, g, a y $\boldsymbol{h}_{R}$}

El índice $h$ está considerado un indicador robusto (Dorta-González y Dorta-González, 2010) porque se basa en la relación cuantitativa que existe entre la productividad de un investigador y el éxito que conceden sus pares a sus propuestas. Sin embargo, no discrimina en niveles intermedios, ya que no tiene en cuenta las citas que recibe un artículo por encima del índice. Es decir, dos autores tendrían un mismo índice $h$ si tienen el mismo número $h$ de artículos con al menos un número $h$ de citas cada uno de 
ellos pero no influyen en esa cuantificación las citas que superan $h$. Por ejemplo, un investigador con 5 artículos con 5 citas cada uno y otro con 5 artículos con 20 citas cada uno tendrían ambos un $h=5$, aunque el valor de impacto absoluto de uno de ellos cuadriplique al otro. Esta falta de discriminación en niveles intermedios también puede interpretarse como un síntoma de que $\mathrm{h}$ es un indicador que promociona a los productores masivos de artículos frente a los productores selectivos de pocos textos pero muy referenciados.

Al índice $h$ se le reprocha, también, que no pueda emplearse para realizar comparaciones entre áreas o entre campos científicos, ya que el número de investigadores y de revistas y las particularidades de producción y difusión científica son diferentes en cada una de ellas. Tampoco se considera un indicador consistente porque un mismo artículo colectivo puede elevar el índice h de alguno de sus firmantes pero dejar igual el de los otros. Para contribuir a corregir estas posibles distorsiones se han establecido otros indicadores de apoyo en la interpretación de $h$, entre los que destacan como más referenciados: mediana, $h 5, i 10, m, h_{R}, g$ y $h$ futuro:

- Índice $h / m e d i a n a$. Refleja las citas que tiene el articulo central del $h$-core o conjunto de artículos que tienen un número de citas igual o superior al número $\mathrm{h}$ del índice h. Es decir, los trabajos que computan para el $h$ del autor o de la revista.

- Índice h5. El índice $h$ limitado a las citas recibidas por los artículos de un autor en los últimos cinco años naturales completos, independientemente del año de publicación del artículo.

- Índice i10. Número de artículos que han recibido al menos diez citas cada uno de ellos. Combinado con $i h 5$ se sabe el número de artículos con diez citas en los últimos cinco años.

- Índice m. (Hirsch, 2005). Es el resultado de dividir h entre el número de años como investigador, contados a partir de la defensa de la tesis doctoral o del primer artículo en una revista científica. Corrige que los investigadores noveles se vean desfavorecidos en el cálculo de $h$.

- Índice $h_{R}$. Es el valor referencial que se espera de $h$ para un área.

- Índice g (Egghe, 2006). Pretende compensar el impacto de las citas de artículos que superan el dígito $h$ del índice $h$ ya que tiene en cuenta de forma acumulativa el impacto o número de citas. Explicado de una manera práctica se obtiene cuando ordenando en una fila todos los artículos de un autor de mayor a menor según el número de citas que cada uno ha recibido. En una segunda columna se anota el valor del cuadrado del dígito que refleja la posición del artículo en la fila $\left(g^{2}\right)$. Y en una tercera se refleja el número de citas acumuladas $\left(\sum h\right)$. El índice $g$ lo determina el puesto que ocupa el último artículo en el que el valor del cuadrado de su posición $\left(g^{2}\right)$ es menor o igual que el número de citas acumuladas $\left(\sum h\right)$.

- Índice futuro (Acuna, 2012). A través de una combinatoria de datos de la actividad científica (número de citas, trabajos publicados...) y basándose en predicciones tomadas de estudios de biología evolutiva, se prevé la evolución del indice $h$ de investigadores que tienen publicaciones en los últimos cinco años. 


\section{Bibliometría en Google Scholar Metrics e impacto en las Ciencias Sociales}

La irrupción de GSM en la bibliometría se ha cuestionado porque se basa en una categorización realizada por Google sin dejar claro el criterio usado para determinar áreas y disciplinas; porque no desvela si ha incluido una revista en varias áreas y, entre otras consideraciones, porque valida todas las citas que detectan sus motores de rastreo. Sin embargo, también se ha considerado una propuesta con futuro: a través de los perfiles individuales y colectivos en abierto se aumenta la visibilidad de los contenidos y se democratiza la difusión de los resultados de investigación al ponerlos al alcance de la sociedad en acceso libre, universal y gratuito (Túñez y De Pablos, 2013).

GS funciona desde 2004 pero empieza a ofrecer sus listados de $h 5$ en abril de 2012 con un primer ranking de revistas en inglés, chino, alemán, portugués, español, francés, italiano, japonés, holandés y coreano. En noviembre, corrige errores e incorpora una lista de revistas por áreas temáticas en inglés. Es el primer listado en firme, que se reactualiza a finales de julio de 2013. GSM indexa revistas que en cinco años han publicado al menos cien artículos y han tenido por lo menos una cita, aunque no aclara las bases a las que acude para otorgar las citas a los artículos.

Este ranking anual aporta perspectivas analíticas en la interpretación de indicadores de producción científica, entre las que destacamos que permite desarrollar análisis comparativos globales del impacto que reciben los artículos en función de la lengua de difusión y que amplía las áreas de aplicación del índice $h$. En nuestro entorno, este aspecto es de gran importancia porque facilita la reinterpretación del peso del español como soporte vehicular de ciencia y permite un análisis en perspectiva iberoamericana del castellano y portugués frente a los que se consideran idiomas dominantes (inglés y chino) y otras lenguas europeas.

El análisis se dibuja necesario si se acude a datos como los manejados en 2013 por el Instituto Cervantes: El español fue la lengua más hablada en twitter (2012) y se coloca como tercer idioma en Internet, tras el inglés y el chino. Según Population Reference Boureau, el 7\% de los 7.057.075.000 habitantes del mundo en 2012 es hispanohablante 500 millones, cifra que da una impresión poderosa del español en el planeta.

El panorama que dibujan estas referencias adquiere matices menos brillantes cuando, como indican Rubio y Urrutia (2011), "el prestigio de una lengua se aprecia por los espacios que ocupa fuera del uso doméstico y en esto el español avanza poco". El uso científico forma parte de ese "fuera del uso doméstico", por eso este trabajo se plantea abordar la exploración de la información que las nuevas herramientas de Google proporcionan en el terreno científico, a través del soporte lingüístico de las publicaciones académicas y sus niveles de impacto medidos en índice $h 5$.

En un principio, el índice $h$ no reparaba en Ciencias Sociales y Humanidades por considerar que no eran ámbitos de aplicación (Imperial y Rodríguez, 2005 y 2007). Aunque Google se califique como "un producto inmaduro, que consta de múltiples limitaciones en su configuración actual para la evaluación de las revistas científicas" también se le reconoce que es "una mina de información científica prodigiosa [...] especialmente de utilidad para los científicos de Humanidades y Ciencias Sociales porque controla como nadie lo ha hecho hasta ahora la literatura no anglosajona y 
aquella transmitida por medios distintos a las revistas científicas, que es la peor controlada por los sistemas de información dominantes en el mundo académico" (Delgado, Orduña-Malea, Marcos, Jiménez-Contreras y Ruiz-Pérez, 2012: 4).

La difusión científica en Ciencias Sociales y en Humanidades aún se vehicula en pocas revistas y con ritmos de revisión muy lentos (De Pablos, Mateos y Túñez, 2013). Como apuntan Delgado et al ${ }^{1}(2014,88)$, ambas áreas aún mantienen preferencia por los libros y las monografías y sus investigadores se decantan por publicar en soportes nacionales y en su misma lengua nativa. "Estas prácticas hacen casi inservibles las bases de datos tradicionales que ofrecen recuentos de publicaciones de citas (Web of Science, Scopus, Psycinfo, etc.). Dichas bases de datos cubren casi exclusivamente artículos de revistas, se orientan fundamentalmente a las disciplinas científico-técnicas y poseen un marcado sesgo anglosajón en cuanto a la procedencia e idioma de los documentos que indizan (Archambault et al., 2006), por lo que no resultan adecuadas dados los hábitos de publicación y citación de los investigadores en ciencias sociales y humanas (Osca-Lluch et al., 2013)" (Delgado et al., 2014: 89).

La propuesta bibliométrica de Google ha sido controvertida desde el principio pero también ha avivado el debate sobre la dependencia de la comunidad investigadora de los criterios de empresas multinacionales que tienen su modelo de negocio en la evaluación de la difusión de la investigación. Esa estrategia de mercado se basa en los datos y en la reputación de las mediciones como referente de calidad que ofrecen Thomson Reuters y Elsevier casi a modo de monopolio internacional pero que se ve amenazado por la estrategia de difusión gratuita de indicadores que realiza Google, otra multinacional, basada en la audiencia del servicio. "El eje de negocio se desplaza de la rentabilidad por coste a la gestión de relaciones como producto, ya que se ofrece gratuidad y acceso libre pero los usuarios se convierten en audiencias apetecibles para clientes que necesiten ejecutar operaciones relacionales on-line para promover productos, ideas, personas, servicios u organizaciones" (Túñez y De Pablos, 2013: 134).

\section{El área de Comunicación: investigadores y revistas}

Aunque el debate sobre el índice $h$ se mantiene en algunas áreas desde que Hirsch lo propone en 2005, la de Comunicación en España no comienza a interesarse por él hasta que en 2012 Google Scholar Metrics irrumpe en competencia directa con Web of Science (WoS, Thomson Reuters) y con Scopus (Elsevier) para ofrecer sus propias mediciones bibliométricas. En foros científicos de Comunicación (exceptuando reuniones de editores de revistas) la primera referencia sobre GSM se da en el IV Congreso Internacional Latina de Comunicación Social, en diciembre de 2012, con la aportación de Sánchez Pita "Actualización del Î́ndice H de las revistas de Comunicación, según Google Scholar Metrics". Las primeras discusiones sobre el indice $h$ en encuentros científicos de Comunicación se hacen esperar hasta mayo de 2013, durante el segundo Congreso de la sección de Métodos de Investigación de la Asocia-

1 Los autores anticipan una herramienta para conocer los indicadores $h$ y $g$ de los investigadores de Ciencias Sociales y Humanidades de universidades públicas, pero el enlace que facilitan remite a una página en construcción (consulta 7 de febrero de 2014). 
ción Española de Investigación en Comunicación (AE-IC), celebrado en el Campus María Zambrano de Segovia de la Universidad de Valladolid los días 2 y 3 de mayo de 2013 (Túñez y De Pablos, 2013).

Aunque el índice $h$ no es un indicador válido para establecer comparaciones entre diferentes áreas, es posible determinar un $h$ referencial $\left(h_{R}\right)$ para cada una de ellas, que siempre estará fuertemente relacionado con el factor de impacto de sus principales revistas. Imperial y Rodríguez-Navarro $(2005,2007)$ proponen la fórmula $\mathrm{h}$ de referencia: $h_{R}=16+11 x I F$ y se decantan por defender la inclusión de la propuesta de Hirsch como criterio de medición de impacto de los investigadores en su carrera universitaria, pero advierten que "este sistema, lamentablemente, no es aplicable, salvo alguna excepción, a las ciencias sociales y a las humanidades, porque estas áreas carecen de bases de datos de revistas suficientemente extensas y por el gran peso que estas áreas tiene la publicación de libros" (2005: 8).

Sin descuidar las advertencias, el índice $h$ referencial en el área de Comunicación en España a partir del índice de impacto de las principales revistas elaborado por el grupo $\mathrm{EC}^{3}$ de la Universidad de Granada estaría entre 17 y 18. Los datos de In-RECS de 2011 ofrecen el factor de impacto de 23 revistas ( 3 de ellas con valor cero); si aplicamos en la fórmula de Imperial y Rodríguez-Navarro con la media de IF de todas las revistas, nos llevaría a un $h$ referencial de 17; si solo tomamos en cuenta las revistas del primer cuartil, sería de 18.

Este $h_{R}$ representa un índice aún distante de la realidad de la mayoría de los investigadores y de las revistas científicas. La revisión a finales de 2013 de perfiles de investigadores miembros de la Asociación Española de Investigación de la Comunicación (AE-IC) ofreció un resultado de un $h$ medio de 4 (Túñez, 2014) ${ }^{2}$. Las revistas con $h$ más alto en España están en torno a 14.

Elsevier, Thomson Reuters y GSM usan universos de referencia diferentes para obtener sus indicadores por lo que el $i h$ de un mismo investigador o un mismo equipo puede representarse con dígitos diferentes según el patrón de búsqueda que se utilice. La comparativa entre Google, Thomson Reuters y Elsevier que Delgado y Repiso (2013: 48) realizan sobre la base del listado de Google de noviembre de 2012 deja clara esa diferencia: Identifican 664 revistas de comunicación en todo el mundo de las que menos de la mitad (277) aparecen la lista de GSM de noviembre 2012, pero en Scopus solo están una de cada cuatro (167) y en WoS no llegan a 2 de cada diez (106).

La supremacía anglosajona en $W o S$ y Scopus queda visiblemente amortiguada en Google y la difusión en español casi se triplica en GSM sobre las otras dos bases. GSM es la que indexa más revistas, de más países y en más idiomas y "mide las revistas de forma muy parecida a los clásicos sistemas de evaluación de revistas (WoS y Scopus), por lo que en líneas generales, y a efectos únicamente de ranking, es una alternativa tan fiable y válida como estos para medir el impacto de las revistas" (Delgado y Repiso, 2013: 51). En español se publican el 13,8\% de las revistas que se in-

${ }^{2}$ Es el resultado de una investigación presentada al Congreso de la AE-IC de 2014 realizada sobre un universo de 646 miembros, una muestra de 267 investigadores, un nivel de confianza del $97 \%$, un margen de error del 5\% y una heterogeneidad del $50 \%$. 
cluyen en listados de GSM, el 5,2\% de las revistas indexadas en Scopus y el 5,6\% de las que están en la base de datos de WoS. En inglés, el 59,7\% de las revistas indexadas por Google, el 88,7\% de las de Scopus y el 86,9\% de las de Web of Science.

El análisis de las revistas de Comunicación está abundantemente analizado en las publicaciones de $\mathrm{EC}^{3}$ de la Universidad de Granada. De sus numerosas aportaciones, nos parecen interesante destacar la actualización en octubre de 2013 de este listado de Comunicación (Repiso y Delgado López-Cózar, 2013). El número de publicaciones ascendió de 223 en 2012 a 354 en 2013, lo que significa un incremento del 58,7\%, con incorporaciones de nuevos títulos de España, Brasil, Portugal, Bolivia, Chile, Colombia y México. De las 354 revistas de Comunicación de todo el mundo, 74 son iberoamericanas (20,9\%). Es significativo que mientras en 2012 en el primer cuartil, solo hay una revista de Iberoamérica, la brasileña Interface-Comunicação, Saúde, Educação, en 2013 ya se incorporan, además de la reseñada, Comunicar y Revista Latina de Comunicación Social a ese primer cuartil dominado por revistas anglófonas, mayoritariamente de EEUU, Reino Unido y Canadá.

Aunque $\mathrm{EC}^{3}$ la incluye en sus listas, Interface-Comunicação, Saúde, Educação no aparece referenciada como tal en Qualis, el listado oficial de la Fundación CAPES (Coordenaçao de Aperfeiçoamento de Pessoal de Nivel Superior) del MEC (Ministerio da Educaçao) por el que se categoriza las revistas en Brasil. El ISSN con el que se representa Interface en la Web, con el subtítulo de Comunicação, Saúde, Educação (1807-5762)3 , conduce en el buscador de Qualis a 13 publicaciones de diferentes áreas de evaluación entre las que no están Comunicación ni Ciencias Sociales, por lo que realmente de Comunicación solo podría hablarse de dos revistas de Iberoamérica en ese primer cuartil: Comunicar y Revista Latina de Comunicación Social.

\section{Métodología}

Este trabajo analiza el peso del idioma como soporte de difusión científica, con especial atención a las revistas de Ciencias Sociales, a partir de la recuperación de todos los listados de las revistas Top 100 de Google (noviembre, 2012; julio, 2013) para convertir esos datos en una 'clasificación mundial' con las citas recibidas por las revistas científicas en cada uno de los idiomas de interés general. Este análisis comparativo se realiza calculando la media universal $(\mu)$, definida como la media aritmética de los índice $h 5$ superior e inferior de las revistas integrantes de cada tabla de las 100 revistas más citadas en una lengua, para disponer de números unificados y comparables. También se realiza un cálculo semejante con la media universal median $(\mu \mathrm{m})$. En esta 'clasificación mundial por idiomas', la media universal se representa con la letra griega $m u, \mu$, y la media universal median, como $m u$ media, $\mu \mathrm{m}$.

\subsection{Población y muestra}

Para la investigación se trabaja directamente sobre el universo de revistas de los listados de GSM, ya que se toman como muestra todas las revistas en español y las otras

3 http://www.interface.org.br/ 
ocho lenguas, consideradas por GSM como las más citadas: cerca de 1.000 revistas en 2012 y casi 900 en 2013. La comparativa propuesta era imposible antes de 2012, al no disponerse de las publicaciones más citadas en lengua inglesa, china, portuguesa, alemana, española, francesa, italiana, japonesa, holandesa y coreana. Del listado de 2013 se cae el coreano: ni siquiera disponía de 100 revistas; es una demostración de la poca información existente hasta entonces, que empieza a depurarse. Se mantiene el holandés, que tampoco llega a la centena de publicaciones.

\subsection{Objetivos e instrumentos}

GSM considera revistas que en los últimos cinco años naturales hayan tenido al menos una cita y publicado como mínimo cien artículos: desprecia revistas irrelevantes y las de baja productividad. Los indicadores que ofrecen las listas de 2012 y 2013 permiten abordar cuestiones que hasta ahora eran imposibles de formular, al no disponer de materiales precisos para hacer un estudio comparativo que permita una lectura del peso de las áreas de conocimiento en la difusión en los idiomas citados. Se trabaja con GSM por su acceso universal y gratuito que traslada la divulgación en soportes científicos a los usuarios de la red frente a otras propuestas de pago.

La información base se trabaja para, con carácter exploratorio, conocer la situación de los idiomas como soporte de difusión de investigación, según el sistema de media universal, $т и(\mu)$; aportar una aproximación comparativa entre lenguas y áreas, realizando una cata de las primeras 10 revistas por idioma; determinar el peso por áreas a través del impacto o distribución de citas por materias de revistas en lengua española y ver la situación de las revistas de Ciencias de la Información-Comunicación.

Se interpretan los indicadores disponibles agrupando las cabeceras en español en seis áreas para evaluar la importancia de las revistas, según sean de Salud, Ciencias Sociales, Ciencias de la Naturaleza, Humanidades, Ciencias y Tecnologías e Ingenierías, aunque se admite que la medición no podrá ser exacta porque toda la comunicación científica no se encuentra en las fronteras de una lengua.

Esta prospección pretende conocer el valor científico real del español, según GSM, al poder compararlo con otros idiomas. Lo basamos en el uso que a los artículos dé cada comunidad, medido por las citas que la colectividad científica concede a los trabajos publicados en su propia lengua y a los soporte de producción más representativos.

\subsection{Hipótesis}

Las hipótesis formuladas son:

H1- Los textos en inglés, lengua franca, están por encima de los demás, entre otros motivos por la presencia de autores de otras lenguas que publican en inglés, además de por la robustez de las ciencias y las tecnologías en el ámbito anglosajón. Se encontrarán valores aproximados para todas las lenguas, excepto el inglés, que estará sobrevalorado al emplearse fuera de sus fronteras naturales.

H2- Del español se mantiene, de forma no científica, que es la más importante en todos los sentidos, tras el inglés y el chino. Se proyecta esta idea al ámbito científico para verificar si le lengua española es soporte de difusión más usado, tras inglés y chino. 
H3- Partimos de otra idea socialmente existente: las revistas de Química y de 'ciencias duras' españolas son las más reconocidas, por encima de las demás.

H4- Las revistas de Información/Comunicación presentan datos discretos, por su juventud y el moderado número de investigadores.

Preguntas que se pretenden responder:

P1- ¿Es posible hacer una clasificación mundial de idiomas basada en las citas que las distintas comunidades científicas hacen de artículos de revistas publicadas en su lengua?

P2- ¿Cuáles son las áreas de conocimiento de las revistas en español más destacadas? ¿Hay paralelismo con otras lenguas? llano?

P3- ¿Dónde se sitúa Comunicación en el panorama mundial de revistas en caste-

\section{Resultados}

Los resultados se dividen en dos apartados: según la lengua de las 100 revistas top en las nueve tablas de GSM y según las áreas de conocimiento representadas en la tabla de revistas en lengua castellana, cuyo resultado se compara -además- con los demás idiomas en una cata de las 10 primeras cabeceras de cada lista.

\subsection{Por idiomas}

Para conocer la situación de los idiomas, se origina la Tabla 1 con las cifras máximas y mínimas del ih5 de las revistas de los listados de GSM. Se opera según el sistema metodológico de media universal $(\mu)$ propuesto en este trabajo. Lo mismo se hace con los datos del h5-median $(\mu \mathrm{m})$, para contrastar si el uso de una u otras cifras alteran la clasificación.

Tabla $1-\mu \& \mu \mathrm{m}$ de idiomas

\begin{tabular}{|c|c|c|c|c|c|c|}
\hline \multirow{2}{*}{$\begin{array}{c}(\mu) \\
\text { Diferencia } \\
\text { entre } \\
\text { mayor y } \\
\text { menor } i h 5\end{array}$} & \multirow[b]{2}{*}{ Idioma } & \multirow{2}{*}{$\begin{array}{l}(\mu \mathrm{m}) \\
\text { Diferencia } \\
\text { entre } \\
\text { mayor y } \\
\text { menor } h 5 \text { - } \\
\text { median }\end{array}$} & \multicolumn{2}{|c|}{$i h 5$} & \multicolumn{2}{|c|}{ h5-median } \\
\hline & & & Mayor & Menor & Mayor & Menor \\
\hline 247 & Inglés & 349 & 349 & 102 & 504 & 155 \\
\hline 33 & $\begin{array}{l}\text { Chino } \\
\end{array}$ & 48 & 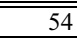 & 21 & 85 & 37 \\
\hline 28 & Portugués & 33 & 38 & 10 & 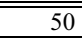 & 17 \\
\hline 15 & Alemán & 17 & 22 & 7 & 28 & 11 \\
\hline 18 & Español & 26 & 28 & 10 & 38 & 12 \\
\hline 12 & Francés & $\overline{6}$ & 18 & 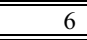 & 19 & 13 \\
\hline$\overline{7}$ & Italiano & $\overline{88}$ & 10 & 3 & 12 & 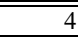 \\
\hline 5 & Japonés & 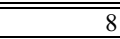 & 9 & 4 & 12 & $\overline{4}$ \\
\hline 9 & Holandés & 11 & 10 & 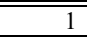 & 12 & 1 \\
\hline
\end{tabular}

Fuente: GSM, 2013. Elaboración propia.

Esta reinterpretación de datos permite observar que las revistas en lengua inglesa reciben un número de citas 7,48 [247/33] veces superior [media universal $\mu$ de 247 
frente a $\mu 33$ del chino] al segundo idioma científico, el chino. La lengua china ocupa la segunda posición, con una $\mu$ de 1,17 [33/28] superior a la lengua portuguesa, que ocupa la tercera posición justo por delante de las revistas en lengua española, que ocupan la cuarta. (Tabla 01).

Con respecto a las demás lenguas europeas, la situación queda en este orden, de acuerdo a la media universal $\mu$ : alemán (15); francés (12); italiano (7) y holandés (9). La importancia de revistas en italiano, japonés y holandés es muy poco significativa, si tenemos en cuenta que en el Top 100 de estos idiomas entran revistas con un ih 5 de 3 (desde la posición 58 de las italianas), de 4 (desde la posición 52 de japonés) y de 4 (las holandesas, con ese índice desde la posición 13 y solamente 79 revistas presentes, lo que advierte -como ya sucedió con el coreano- que el idioma holandés se pueda caer en 2014.

La lista por idiomas según su importancia científica por las citas que reciben las 872 revistas listadas por GSM 2013, reflejadas en la media universal $\mu$ índice-h5 y media universal h5-median, $\mu \mathrm{m}$, queda de esta manera: $\left.1^{\circ}\right)$ inglés ( $\mu 247$ y $\left.\mu \mathrm{m} 349\right)$; $\left.2^{\circ}\right)$ chino $(\mu 33$ y $\left.\mu \mathrm{m} 48) ; 3^{\circ}\right)$ portugués $(\mu 28$ y $\left.\mu \mathrm{m} 33) ; 4^{\circ}\right)$ español $(\mu 18$ y $\mu \mathrm{m} 26)$; $5^{\circ}$ ) alemán $(\mu 15$ y $\left.\mu \mathrm{m} 17) ; 6^{\circ}\right)$ francés $(\mu 12$ y $\left.\mu \mathrm{m} 6) ; 7^{\circ}\right)$ holandés $(\mu 9$ y $\left.\mu \mathrm{m} 11) ; 8^{\circ}\right)$ italiano $(\mu 7$ y $\left.\mu \mathrm{m} 8) ; 9^{\circ}\right)$ japonés $(\mu 5$ y $\mu 8)$. En la presentación de idiomas que hace GS, debería poner al alemán tras el castellano y el holandés por encima de italiano y japonés, aunque en neerlandés no se llega a 100 las revistas del "top 100", lo cual es una incoherencia, que igual hace que Holanda no vuelva a aparecer en este tipo de listados (el corte a una cita x cinco años se hace en el puesto 79) ${ }^{4}$ como pasó de 2012 a 2013 con el coreano, que tampoco llegaban a 100 revistas con al menos una cita en cinco años y 100 papers publicaos en un lustro.

\subsection{Por áreas o campos de conocimiento}

Se confecciona la Tabla 2 con las 198 revistas (hay un título repetido cada año: $R e$ vista iberoamericana de ciencia, tecnología y sociedad y Revista Interuniversitaria de Formación del Profesorado $)^{5}$ en lengua española de 2012 y 2013, distribuidas en nueve grupos, que en ocasiones son interdisciplinares: Salud, Educación, Economía, Ciencias de la Tierra, Ciencias de la Información / Comunicación; Sociología, Ingenierías, Ciencias 'clásicas' y Humanidades. Mediante ese análisis de comprobarán las altas y las bajas que pudieran haber en cada una.

Tabla 2 - Áreas de revistas en español

\begin{tabular}{|c|c|c|c|c|c|}
\hline \multirow{2}{*}{ Áreas } & \multicolumn{2}{|c|}{ Revistas 2012} & \multicolumn{2}{|c|}{ Revistas 2013} & \multirow{2}{*}{ Tendencia } \\
\hline & número & $\%$ & número & $\%$ & \\
\hline Salud & 52 & $52,53 \%$ & 49 & $49,50 \%$ & $-3,03 \%$ - \\
\hline Educación & 14 & $14,14 \%$ & 19 & $19,19 \%$ & $+5,05 \%$ - \\
\hline $\begin{array}{l}\text { Ciencias de la Tierra } \\
\text { (Biología, Oceanografía, } \\
\text { Geología...) }\end{array}$ & 8 & $8,08 \%$ & 9 & $9,09 \%$ & $+1,09 \%$ \\
\hline
\end{tabular}

${ }^{4} \mathrm{http}: / /$ scholar.google.com/citations?view_op=top_venues\&hl=es\&vq=nl [23/02/2014]

5 Por esa razón, la suma de cada año da 99. 


\begin{tabular}{|c|c|c|c|c|c|}
\hline Economía & 8 & $8,08 \%$ & 3 & $3,03 \%$ & $-4,97 \nabla$ \\
\hline Sociología & 6 & $6,06 \%$ & 11 & $11,11 \%$ & $+5,05 \%$ • \\
\hline $\begin{array}{l}\text { Documentación, } \\
\text { Comunicación, } \\
\text { Información }\end{array}$ & 5 & $5,05 \%$ & 6 & $6,06 \%$ & $+1,01 \%$ \\
\hline Ingenierías, Tecnologías & 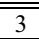 & $3,03 \%$ & 2 & $2,02 \%$ & $-1,01 \% \nabla$ \\
\hline $\begin{array}{l}\text { Ciencias (Matemáticas, } \\
\text { Químicas...) }\end{array}$ & 1 & $1,01 \%$ & & $0 \%$ & $-1.01 \%$ \\
\hline Humanidades & 2 & $2,02 \%$ & 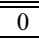 & $0 \%$ & $-2,02 \% \vee$ \\
\hline TOTAL & 99 & $100,00 \%$ & 99 & $100 \%$ & \\
\hline
\end{tabular}

Fuente: GSM. Elaboración propia.

Los hallazgos más notables son:

Las revistas de Salud (Medicina, Psicología) dominan con un 52,53\% en 2012 y un $49,50 \%$ en 2013 . Son revistas asentadas y con ligeras variaciones en sus índices, a pesar de que algunas revistas se han caído de la tabla. Estas bajas son pocas (tendencia negativa del 3,03\%).

En segundo lugar aparecen las revistas de Educación, con el 14,14\% y el 19,19\%, respectivamente, y un aumento de 5 cabeceras, lo que significa una tendencia al alza del $5,05 \%$.

El tercer y cuarto lugar lo disputan, respectivamente, las revistas Ciencias de la Tierra (Biología, Oceanografía, Geología) y de Economía, que si en 2012 suponían el 8,08\%, en 2013 significan el 9,09\% y el 3,03\%, debido, sobre todo, a la caída de revistas de Economía, que de 8 han pasado a 3, con una tendencia de $-4,97 \%$, mientras el repunte de Ciencias de la Tierra es de 1,09\%.

El quinto lugar lo ocupan las revistas de Sociología, que de 6 en 2012 han pasado a 11 en 2013 (incremento notable del 11,11\%) y un tendencia al alza del 5,05\%.

Las revistas con contenido total o parcial del área de Comunicación ocupan el sexto lugar: 4 en 2012 y una más, 5, en 2013, lo que supone el 6,06\% del Top 100, todas en fase ascendente. (Tabla 3 ).

Tabla 3. Revistas de Comunicación en español

\begin{tabular}{|c|c|c|c|c|}
\hline \multirow{2}{*}{ Revista } & \multicolumn{2}{|c|}{2012} & \multicolumn{2}{|c|}{2013} \\
\hline & Situación & h5/ h5-med & Situación & $\begin{array}{c}h 5 / h 5 \\
\text { med }\end{array}$ \\
\hline EPI & 26 & $14 / 17$ & $15 \wedge$ & $\begin{array}{ll}16 / 21 \bullet \\
\end{array}$ \\
\hline Comunicar & No & No & $40 \wedge$ & $\begin{array}{ll}13 / 16 \bullet \\
\end{array}$ \\
\hline RLCS & 96 & $9 / 17$ & $45 \wedge$ & 12/19 ^ \\
\hline Pixel-Bit & 82 & $10 / 13$ & $49 \wedge$ & $\begin{array}{l}12 / 17 \bullet \\
\end{array}$ \\
\hline Telos & 86 & $10 / 13$ & $70 \wedge$ & 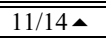 \\
\hline
\end{tabular}

Fuente: GSM. Elaboración propia.

Para hacer una aproximación comparativa entre lenguas elaboramos las tablas 4 y 5 con la agrupación de revistas por grandes áreas de conocimiento y una cata de las primeras 10 de cada idioma en 2013. En estas revistas de impacto máximo, el dominio de contenidos de Salud es abrumador, seguido de Ciencias Sociales (Tabla 4). Por 
idiomas, Salud también es el área con más divulgación científica en todos los casos, excepto en italiano, que es mayoritariamente de Ciencias Sociales; en japonés priman temas de Tecnología e Ingeniería; en alemán casi es igual Salud y Ciencias Sociales; y en chino Salud, Ciencias Sociales y Tecnologías se equilibran (Tabla 5).

Tabla 4. Revistas por grandes áreas de conocimiento

\begin{tabular}{|c|c|c|c|c|c|}
\hline Rama & 2012 & 2013 & $\% 2012$ & $\% 2013$ & Evolución \\
\hline Salud & 52 & 49 & $52,55 \%$ & 499,49 & $-3,06 \nabla$ \\
\hline Ciencias Sociales & 33 & $\overline{39}$ & $33,33 \%$ & 39,39 & $+6,06 \wedge$ \\
\hline Ciencias de la Naturaleza & 8 & 9 & $8,08 \%$ & 9,09 & $+1,01 \bullet$ \\
\hline Humanidades & 2 & - & $2,02 \%$ & - & $\nabla$ \\
\hline Ciencias & 1 & - & $1,01 \%$ & - & $\nabla$ \\
\hline Tecnologías / Ingenierías & 3 & 2 & $3,03 \%$ & 2,02 & $-1,01 \vee$ \\
\hline
\end{tabular}

Fuente: GSM. Elaboración propia.

Tabla 5. Top-10 por grandes áreas de conocimiento

\begin{tabular}{|l||c||c|c|c|c|c|c|c|c||}
\hline & Inglés & Chino & Portugués & Español & Alemán & Francés & Italiano & Japonés & Holandés \\
\hline \hline Salud & $\mathbf{8}$ & $\mathbf{3}$ & $\mathbf{8}$ & $\mathbf{9}$ & $\mathbf{5}$ & $\mathbf{5}$ & 1 & 1 & $\mathbf{9}$ \\
\hline \hline CCSS & - & 2 & - & 1 & 4 & 3 & $\mathbf{7}$ & - & 1 \\
\hline \hline CCNN & 2 & 1 & 2 & - & & - & 1 & 1 & - \\
\hline \hline TT/II & - & $\mathbf{3}$ & - & - & - & - & - & $\mathbf{8}$ & - \\
\hline
\end{tabular}

Fuente: GSM-2013. Elaboración propia.

\section{Conclusiones}

Al ser el inglés el idioma franco, citas y autores no son necesariamente de países de lengua inglesa, por lo que habrá "préstamos", como es natural en una lengua universal. El hecho de que sea la lengua científica por antonomasia aumenta la distancia con los otros idiomas, con indicador de citación seis veces superior a la segunda lengua, el chino.

La emergencia de la economía china va aparejada con el fortalecimiento del idioma. Queda patente que China no es solamente un país de recepción de ciencia y técnica occidentales. Ninguna de las 100 revistas chinas está titulada en inglés (Alemania tiene dos; Holanda, una y hay cinco entre las revistas basadas en países hispanohablantes).

El portugués se ve fortalecido al situarse como tercera lengua científica mundial, con 40 cabeceras que en el título llevan alusión a Brasil, incluso 22 de ellas se llaman Revista Brasileña de... Tras la lengua portuguesa aparece la española. La aplicación de $\mu$ y $\mu \mathrm{m}$ revalidan la hipótesis primera y refutan la segunda: es posible originar una clasificación global por idiomas y dan respuesta positiva a la primera de las preguntas de investigación.

Los listados de revistas en español presentan estabilidad en su cabecera: se mantienen las cuatro primeras revistas, una mexicana y tres españolas, una de éstas en colaboración con una asociación colombiana, editada por Elsevier desde 2013. De las 99 revistas, 68 se mantienen en la segunda actualización y 20 ascienden en la tabla, algunas de nueva entrada, con una caída de 11 cabeceras. Destacan con sensible dife- 
rencia las de Salud, con relevancia semejante a las revistas en inglés, chino, portugués, alemán y francés. En japonés prevalecen las de tipo técnico y en italiano las de Ciencias Sociales (Economía y Trabajo). En italiano y japonés la relevancia es muy baja. En holandés ni siquiera hay 100 revistas referenciadas. Queda descartada la segunda hipótesis y resuelta la segunda pregunta.

Las revistas de Información-Comunicación presentes en las listas han subido de cuatro a cinco, si incluimos las dos revistas relacionadas con Educación y Comunicación/Información. El sector mantiene una discreta presencia, con un ascenso genérico de 28,7 puntos, desde la posición media en 2012 de 72,56 a una posición media en 2013 de 43,8. ${ }^{7}$ Se corrobora la hipótesis 4 y se resuelve la tercera pregunta: El Profesional de la Información pasa del puesto 26 al 15; Revista Latina de Comunicación Social, del 96 al 45; Pixel, del 82 al 49 y Telos, del 86 al 70. Se incorpora Comunicar, en el 40.

\section{Referencias bibliográficas}

ACUNA, Daniel E.; ALLESINA, Stefano; \& KORDING, Konrad P. (2012): "Future impact: Predicting scientific success". Nature, vol. 489, pp. 201-202 [doi:10.1038/489201a]

BORDONS, María; FERNÁNDEZ, María Teresa; y GÓMEZ, Isabel (2002): “Advantages and limitations in the use of impact factor measures for the assessment of research performance in a peripheral country". Scientometrics, 53, pp. 195-206.

COSTAS, Rodrigo y BORDONS, María (2007): "Una visión crítica del índice h: algunas consideraciones derivadas de su aplicación práctica". El profesional de la información, $\mathrm{n}^{\mathrm{o}}$ 5, 2007, pp. 427-432. http://dx. [doi.org/10.3145/epi.2007.sep.04]

DE PABLOS COELLO, José Manuel (2013): "Contra Google todo vale. ¿Puede la academia esperar algo de Google Scholar?". Actas do XIII Congreso Internacional IBERCOM. Comunicación, Cultura e Esferas de Poder, pp. 1727-1742. $\mathrm{http} / / / \mathrm{www}$.estudosaudiovisuais.org/lusofonia/revision/ActasXIIICongresoIBERCOM.pdf

DE PABlos COELLO, José Manuel; MATEOS MARTíN, Concha; y TÚÑEZ LÓPEZ, Miguel (2013): "Google, cambia el paradigma de la métrica científica". Historia y Comunicación Social. Vol. 18. (Especial Diciembre), pp. 225-235. Madrid, Servicio de Publicaciones de la Universidad Complutense. DOI: [http://dx.doi.org/10.5209/rev_HICS.2013.v18.44327]

DELGADO LÓPEZ-CÓZAR, Emilio y REPISO CABALLERO, Rafael. (2012): “Índice $\mathrm{H}$ de las revistas de Comunicación según Google Scholar Metrics (20072011)", EC Miscelánea. http://hdl.handle.net/10481/22483

$626+96+82+86 / 4=72,5$.

$715+40+45+49+70 / 5=43,8$. 
DELGADO LÓPEZ-CÓZAR, Emilio y CABEZAS-CLAVIJO, Álvaro (2012): “Google Scholar Metrics revisado: Ahora empieza a ir en serio". En $\mathrm{EC}^{3}$ Working Papers, p. 8.

DELGADO LÓPEZ-CÓZAR, Emilio y REPISO CABALLERO, Rafael (2012): “Índice $\mathrm{H}$ de las revistas de Comunicación según Google Scholar Metrics (20072011)". En EC ${ }^{3}$ Miscelánea.

DELGADO LÓPEZ-CÓZAR, Emilio y REPISO CABALLERO, Rafael (2013): “El impacto de las revistas de comunicación: comparando Google Scholar Metrics, Web of Science y Scopus". En Comunicar no 41, v. XXI, p. 45-52. [http://dx.doi.org/10.3916/C41-2013-04]

DELGADO LÓPEZ-CÓZAR, Emilio; ORDUÑA-MALEA, Enrique; MARCOS CARTAGENA, Diego; JIMÉNEZ-CONTRERAS, Evaristo; y RUIZ PÉREZ, Rafael (2012): "Journal Scholar: Una alternativa internacional, gratuita y de libre acceso para medir el impacto de las revistas de Arte, Humanidades y Ciencias Sociales". EC $C^{3}$ Working Papers 5. http://digibug.ugr.es/handle/10481/20375

DELGADO LÓPEZ-CÓZAR, Emilio; ORDUÑA-MALEA, Enrique; MARCOS CARTAGENA, Diego; JIMÉNEZ-CONTRERAS, Evaristo; y RUIZ-PÉREZ, Rafael (2007). "El factor de impacto de las revistas científicas: limitaciones e indicadores alternativos". El profesional de la información, vol.16 (1), p. 4-11.

DELGADO LÓPEZ-CÓZAR, Emilio; ORDUÑA-MALEA, Enrique; JIMÉNEZCONTRERAS, Evaristo;y RUIZ-PÉREZ, Rafael (2014): "H index Scholar: el índice de los profesores de las universidades públicas españolas en humanidades y ciencias sociales". El Profesional de la Información, enero-febrero, v.23 (1), 987 94. [http://dx.doi.org/10.3145/epi.2014.ene.11]

DELGADO LÓPEZ-CÓZAR, Emilio; AYLLÓN, Juan Manuel; y RUIZ-PÉREZ, Rafael (2013): "Índice H de las revistas científicas españolas según Google Scholar Metrics (2007-2011)", EC Informes, $\mathrm{n}^{\mathrm{0}} 3$.

DELGADO LÓPEZ-CÓZAR, Emilio; MARCOS CARTAGENA, Diego; JIMÉNEZCONTRERAS, Evaristo; y RUIZ-PÉREZ, Rafael (2013): "Índice H de las revistas españolas de Ciencias Sociales y Jurídicas según Google Scholar (2002-2011)”. $E C^{3}$ Informes, $\mathrm{n}^{\circ} 4$.

DORTA-GONZÁLEZ, Pablo y DORTA-GONZÁLEZ, María Isabel (2010): “Indicador bibliométrico basado en el índice h". Revista Española de Documentación Cientifica, vol. 33, pp. 225-245. [http://dx.doi.org/10.3989/redc.2010.2.733]

EGGHE, Leo (2006): “Theory and practise of the g-index". Scientometrics, vol. 69 (1), p. 131-162.

GRUPO SCIMAGO (2006): "El índice $h$ de Hirsch: aportaciones a un debate". $E l$ profesional de la información, vol. 15, pp. 304-306. [http://dx.doi.org/10.3145/epi.2006.jul.08] HIRSCH, Jorge (2005): “An index to quantify an individual's scientific output". Proceedings of the National Academy of Sciences of the United States of America, vol. 102, pp. 16569-16572. [http://dx.doi.org/10.1073/pnas.0507655102] 
HIRSCH, Jorge E. (2012): “An index to quantify an individual's scientific research output that takes into account the effect of multiple coauthorship". Scientometrics, vol. 85, pp. 741-754.

IMPERIAL, Juan y RODRÍGUEZ-NAVARRO, Alonso (2005): "Utilidad del índice h de Hirsch para evaluar la investigación en España". En: http://www.bit.etsia.upm .es/Imperial_Rodriguez-Navarro.pdf

IMPERIAL, Juan y RODRÍGUEZ-NAVARRO, Alonso (2007): "Usefulness of hirsch's h-index to evaluate scientific research in Spain". Scientometrics, vol. 71, $\mathrm{n}^{\circ} 2$, pp. 271-282. [http://dx.doi.org/10.1073/pnas.0507655102]

POPULATION REFERENCE BOUREAU (PRB) http://www.prb.org/pdf12/2012population-data-sheet_spanish.pdf

REPISO, Rafael y DELGADO LÓPEZ-CÓZAR, Emilio (2013): “H Index Communication Journals according to Google Scholar Metrics (2008-2012)". EC ${ }^{3}$ Reports, 6: 26 octubre 2013. http://ec3noticias.blogspot.com.es/2013/10/publicado-el-indice-h-de-las-revistas.html

RUBIO, Fanny y URRUTIA, Jorge (2011): "La debilidad del español", El País, 25/03/2011. http://elpais.com/diario/2011/03/25/opinion/1301007608_850215.html

SÁNCHEZ PITA, Fernando (2012): “Actualización del Índice H de las revistas de Comunicación, según Google Scholar Metrics". Actas IV Congreso Int. Latina de Comunicación Social. La Laguna, http://www.revistalatinacs.org/12SLCS /2012_actas/209_Pita.pdf [04-09-2013]

SILVA AYÇAGUER, Luis Carlos (2012): "El Índice-h y Google académico: una simbiosis cienciométrica inclusiva". Acimed, vol. 23, n 3, pp. 308-322. http://acimed .sld.cu/index.php/acimed/article/view/384

TÚÑEZ LÓPEZ, Miguel (2012): La gestión de la comunicación en las organizaciones. Sevilla/Zamora, Comunicación Social Ediciones.

TÚÑEZ LÓPEZ, Miguel (2013): “El 'índice h' de la investigación en Comunicación en España, Portugal y Latinoamérica: Web of Knowledge (WoK), Scopus y Google Scholar Metrics". Communication and Society/Comunicación y Sociedad, vol. XXVI, n. 4, pp.53-75. http://www.unav.es/fcom/comunicacionysociedad/es/resumen.php?art_id=469 [31-10-2013]

TÚÑEZ LÓPEZ, Miguel (2014): "El índice h como indicador de calidad de la difusión de la investigación en Comunicación en España: perfiles personales en Google Scholar Metrics. IV Congreso de la Asociación Española de Investigación de la Comunicación AE-IC Espacios de Comunicación. Bilbao, 2014. http://www.aeic2014bilbao.org/

TÚÑEZ, Miguel y DE PABLOS, José Manuel (2013): “El 'índice h' en las estrategias de visibilidad, posicionamiento y medición de impacto de artículos y revistas de investigación". Actas del II Congreso Nacional sobre Metodología de la Investigación en Comunicación y del Simposio Internacional sobre Política Científica en 
Comunicación, Universidad de Valladolid, Segovia, mayo 2013, pp. 133-150, http://uvadoc.uva.es/handle/10324/2996 [04-09-2013] 